\title{
El Proyect3ES como metodología transdisciplinar de aprendizaje por proyectos en el Grado de Educació Social. Plan de evaluación y valoración del alumnado ${ }^{1}$
}

\author{
Bellver Moreno, María Carmen ${ }^{\text {a }}$ Bakieva, Margarita ${ }^{b}$; De Ramón Felguera, Davinia ${ }^{c}$ \\ ${ }^{a}$ Profesora Contratado Doctor del Departamento de Teoría de la Educación. Universitat de València. \\ m.carmen.bellver@uv.es ${ }^{b}$ Profesora Ayudante Doctor del Departamento de Métodos de Investigación y Diagnóstico \\ en Educación. Universitat de València, margarita.bakieva@uv.es y ${ }^{\mathrm{c}}$ Profesora asociada del Departamento de \\ Educación Comparada e Historia de la Educación. Universitat de València, davinia.ramon@uv.es
}

\begin{abstract}
This paper presents a specific teaching strategy for higher education, based on the interdisciplinarity of subjects and the design of an inter-area intervention project. It is aimed at students of the Degree in Social Education, from the University of Valencia, and seeks that students can can build their learning autonomously, in work teams, from several subjects simultaneously by design of an intervention project that responds to some educational and acutal need identified in context. From the presentation, the work is focused on the evaluation process and on the instruments that have been used. An evaluation plan is presented by the information retrieved from students, teachers and external organizations to reflect on the project as a teaching strategy. The evaluation system is presented, combining qualitative and quantitative data taking into account the different types of evaluation (context, input, process and product). Specifically, the results of the final evaluation of the students presented by average ratings of a closed-question questionnaire. The results show the general satisfaction of the students with the project, highlighting the link with the professional field as an especially positive element, and the need to reinforce the initial presentation of the project as an element to improve.
\end{abstract}

Keywords: transdisciplinarity, teaching coordination, university teaching staff, evaluation, project learning, cooperative learning.

\section{Resumen}

Se presenta una estrategia didáctica específica para educación superior, basada en la interdisciplinariedad de asignaturas y el diseño de un proyecto de intervención interáreas. Está dirigida al alumnado del Grado de Educación Social, de la Universidad de Valencia, y busca que el alumnado construya, de manera autónoma, en equipos de trabajo, abordando desde varias asignaturas simultáneamente, el diseño de un proyecto de intervención, que responda a una necesidad real identificada en el contexto. A partir de la presentación, se centra el trabajo en el proceso de evaluación y en los instrumentos que se han utilizado. Se presenta un plan de evaluación que recoge información de alumnado, profesorado y organizaciones externas para

\footnotetext{
${ }^{1}$ Proyecto seleccionado y financiado en el Programa PID: Proyectos de Innovación Docente 2019-2020. Universitat de València (código UV-SFPIE_PID19-1098202).
} 
reflexionar respecto al proyecto como estrategia didáctica. Se presenta el sistema de evaluación, con datos tanto cualitativos como cuantitativos teniendo en cuenta los diferentes tipos de evaluación (evaluación de contexto, de entrada, de proceso y de producto). De manera específica, se muestran los resultados de la evaluación final del alumnado recogidos mediante un cuestionario de preguntas cerradas. Los resultados evidencian la satisfacción general del estudiantado con el proyecto, destacando la vinculación con el ámbito profesional como elemento especialmente positivo, y la necesidad de reforzar la presentación inicial del proyecto como elemento a mejorar.

Palabras clave: transdisciplinariedad, coordinación docente, profesorado universitario, evaluación, aprendizaje por proyectos, aprendizaje cooperativo.

\section{Introducción}

El concepto de innovación docente dentro del ámbito universitario debe vincularse con la transferencia del conocimiento y con cambios en los procesos del sistema formativo. En los últimos años en las universidades se ha apostado por la innovación, creando servicios y vicerrectorados vinculados con la misma, aunque en la mayoría de los casos relacionada con cambios metodológicos relacionados con el EEES y el soporte del uso de las TIC's en la formación (Gros y Lara, 2009). En todo proceso de innovación hay que incidir especialmente en que se sientan partícipes del mismo tanto los profesores/as como los alumnos/as.

La universidad es una de las mayores instancias productoras de conocimiento, unido a un destacado rol como motor de cambio social y de futuro, que debe priorizar la formación de profesionales versátiles y eficaces en el ámbito universitario, con capacidad inventiva y de amplia visión de futuro y con herramientas para la innovación y la creatividad para afrontar retos en su vida personal y profesional (Alfonso-Benlliure y Bellver, 2020).

Por tanto, la enseñanza superior debe primar la creación de entornos y experiencias que permitan al alumnado construir conocimiento por sí mismo y crear comunidades de aprendices que descubran y resuelvan problemas (Gargallo, 2016) de manera innovadora. Un medio a nuestro alcance es mediante la interdisciplinariedad de asignaturas, puesto que "urge definir más proyectos colegiados a fin de que nuestro alumnado despliegue sus capacidades y mejore su autonomía de acción en torno a cuestiones o problemas fundamentales de su interés científico y profesional" (Santos, 2016, p.18).

Así, el proceso de innovación docente en la universidad, debe concebir el proceso de enseñanzaaprendizaje, con un marcado carácter profesionalizador, debe impregnarse de connotaciones prácticas y desarrollar en el alumnado competencias clave, tales como son: la resolución de problemas en el medio real, la capacidad de reflexión, el pensamiento crítico, la capacidad de autoaprendizaje, la capacidad de adaptación, la asunción de riesgos, la colaboración, el carácter emprendedor y la creatividad, competencias todas ellas propias de la formación del futuro educador/a social.

Esta construcción de contextos educativos orientados hacia la promoción de la innovación en las aulas universitarias debe contemplar los siguientes ejes (Verde y Bellver, 2020): a) más trabajo interdisciplinar, partiendo de la noción que los contenidos se interrelacionan permanentemente "es espiral" y así debería ser considerado en los diseños curriculares de las asignaturas; b) concepción distinta del contexto de enseñanza-aprendizaje donde imperen adjetivos como motivación, interés, pasión, placer puesto que son 
palabras que se relacionan directamente con la creatividad y la innovación y c) docentes innovadores que propongan nuevas estrategias metodológicas y de aprendizaje para su alumnado.

\section{Objetivos}

El objetivo general del proyecto de innovación que se presenta en esta comunicación es facilitar un aprendizaje interdisciplinar, significativo, fundamentado y vinculado con la práctica profesional, como se presentará en el siguiente apartado.

En concreto, los objetivos específicos de Project3ES son:

1) Facilitar la transdisciplinariedad del aprendizaje al ámbito de la Educación Social, visibilizando la complementariedad de perspectivas que las diferentes asignaturas ofrecen para analizar un contexto socioeducativo.

2) Diseñar, elaborar, planificar y evaluar un proyecto de intervención socioeducativo en grupo, a partir del análisis histórico, político y teórico de una determinada temática y de un adecuado análisis de las necesidades y del contexto.

3) Desarrollar técnicas conscientes, explícitas, estructuradas y consensuadas de trabajo en grupo, para desarrollar un aprendizaje colaborativo que sea significativo.

4) Visibilizar y tomar conciencia del aprendizaje significativo ofrecido al Grado de Educación Social, a partir de su vinculación con problemáticas reales, de tal forma que el proyecto planteado sea construido a partir de una necesidad real identificada al contexto, con la participación de los implicados.

5) Incorporar estrategias significativas de evaluación del proceso de trabajo a los grupos y de los resultados ofrecidos con los proyectos, haciendo atención que esta evaluación sea cuidadosamente formativa, además de cumplir su función sumativa.

6) Difusión de la propuesta y sus resultados dentro de la comunidad universitaria (dentro y fuera de la Universitat de València) para visibilizar la factibilidad y el valor formativo de estas estrategias colaborativas, y poder mejorarla a partir de las aportaciones de los otros.

En esta comunicación, de una forma especialmente clara, se pretende presentar el proceso de evaluación seguido, con sus diferentes técnicas de recogida de información y fuentes, desde la perspectiva de complementariedad metodológica.

\section{Desarrollo de la innovación.}

\subsection{EI Proyecto Project3ES.}

El alumnado del Grado de Educación Social, en los cuatro años de formación, debe obtener herramientas socioeducativas para desarrollar su diversificada actividad profesional, que comprende desde la infancia hasta las personas mayores, incluyendo centros de menores, centros naturales de ocio y tiempo libre, escuelas de adultos, prisiones, residencias, centros de día, instituciones comunitarias dedicadas a la prevención y a la reinserción de personas drogodependientes, etc.; priorizando para ello una formación práctica de los conocimientos adquiridos, con metodologías activas y participativas, centradas en la realidad social.

La propuesta de innovación que presentamos desde el Grado de Educación Social de la Universidad de Valencia, nace con la finalidad de aportar al alumnado estas herramientas socioeducativas necesarias para su desarrollo profesional en un medio real (el alumnado diseña un Proyecto de intervención en una entidad o recurso real), y en aras de responder a las demandas del alumnado del grado de no realizar 
trabajos inconexos y aislados, planteando un trabajo interdisciplinar y coordinado entre todas las asignaturas del primer cuatrimestre de tercer curso (Perales, Conchell, De Fez, Bellver y Horcas, 2020).

Así, el proyecto consiste en coordinarse desde estas materias que se imparten simultáneamente, y establecer un único trabajo para las cinco asignaturas, a elaborar en grupos de 4 estudiantes. El trabajo integra distintas partes y recoge todo lo trabajado por el mismo alumnado durante cuatro meses. Las asignaturas implicadas son:

- Historia de la Educación Social en España

- Política de la Educación Social

- Educación, Participación Social, Desarrollo Comunitario e Inclusión

- Intervención Educativa en Procesos de Adaptación Social

- Evaluación de Programas e Instituciones Socioeducativas

A este tarea común se le ha denominado 'Trabajo Grupal Interáreas', de modo que se identifique claramente que se trata de una propuesta conformada por las distintas materias, se trata, en definitiva, de diseñar un programa de intervención que contemple las distintas perspectivas: análisis del contexto y del colectivo, perspectiva histórica y política, marco teórico de referencia, estructuración del programa y su evaluación.

Así, la originalidad del proyecto reside fundamentalmente en: a) la transversalidad puesto que implica cinco asignaturas de un primer cuatrimestre con lo que la interdisciplinariedad también está asegurada, b) la implicación de todo el profesorado implicado desde el acuerdo democrático y el compromiso colectivo y c) la vinculación con las necesidades del contexto, y con el futuro desarrollo profesional de nuestro alumnado.

El objetivo principal del desarrollo del Proyecto de innovación sería entender que la finalidad de la formación universitaria debe ser priorizar la formación de profesionales versátiles y eficaces con capacidad inventiva y de amplia visión de futuro y con herramientas para la innovación y la creatividad para afrontar retos en su vida personal y profesional (Alfonso-Benlliure y Bellver, 2020). Esta finalidad práctica de la formación universitaria es el motor del Proyecto de innovación que presentamos, y se ha concretado en los objetivos planteados en el apartado anterior.

La metodología del proyecto de innovación desarrollado tiene los siguientes ejes: a) coordinación docente; b) aprendizaje basado en proyectos; c) aprendizaje cooperativo; y d) aprendizaje basado en problemas.

Esta estrategia metodológica se encuentra muy extendida entre los distintos niveles educativos en los últimos años. Consiste en el trabajo autónomo por parte del alumno/a, para resolver un problema complejo planteado por el/a docente, contando con su guía y asesoramiento. Suscita la participación activa del estudiante en la resolución colectiva de un problema relevante, que curricularmente es significativo e interdisciplinar (Font Ribas, 2004). Los/as estudiantes deben tomar la responsabilidad de su propio aprendizaje, identificando lo que necesitan conocer para tener un mejor entendimiento y manejo del problema en el cual están trabajando, y determinando dónde conseguir la información necesaria (libros, revistas, profesores, internet, etc.). El/a docente se convierte en consultor del estudiantado (Morales y Landa, 2004).

La combinación de metodologías, el trabajo coordinado del equipo docente, la participación del estudiantado del curso anterior, y la experiencia acumulada en los cursos precedentes ofrecen garantías de la viabilidad de la propuesta. La propuesta de construir una visión interdisciplinar respecto a un ámbito concreto de la educación social, en combinación de asignaturas teóricas y prácticas, entendemos que 
desarrolla un trabajo holístico que podrá transferir a otras asignaturas del grado y en concreto al TFG (Trabajo Fin de Grado).

Así el "Trabajo Grupal Interáreas" lo venimos desarrollando desde hace varios cursos en un principio entre dos profesoras, para posteriormente ir implicando al resto de profesorado. En el curso 2019/20 se presenta formalizado como proyecto de innovación docente en el que participamos 7 profesoras y 4 profesores del primer cuatrimestre del Grado de Educación Social (Proyecto seleccionado en el Programa PID: Proyectos de Innovación Docente 2019-20020. Universitat de València, código UV-SFPIE_PID191098202). Es en este curso escolar donde hemos realizado una evaluación del proyecto involucrando tanto al profesorado como al alumnado del Grado.

En ese trabajo, no obstante, nos centraremos únicamente, por motivos evidentes de espacio, en la valoración final de estudiantado al cuestionario de preguntas cerradas.

\subsection{El diseño de la evaluación del Proyecto Project3ES.}

Para la evaluación del proyecto se ha recogido información de estudiantado y profesorado, en diferentes momentos del desarrollo del mismo, como se ha presentado en otra comunicación en este mismo Congreso (Perales, Conchell y Lorente, 2020).

De hecho, la evaluación del proyecto interáreas ha acompañado todo el proceso de desarrollo del mismo, desde la orientación de evaluación de programas (Martínez Mediano, 2007, Pérez Juste, 2014) recorriendo los diferentes tipos de evaluación propuestos por Stufflebeam (1987), desde la interpretación ajustada a criterios de evaluación (Perales, Ortega y Jornet, 2011).

Para el diseño de los instrumentos de recogida de información se han consultado experiencias previas que aportaban alguna información en este sentido. Maldonado (2009, p. 173), en su propuesta sobre Aprendizaje basado en Proyectos Colaborativos, habla de la observación, la entrevista y los testimonios focalizados, para recoger información del estudiantado, pero no ofrece datos específicos sobre los instrumentos. Por otro lado, Guerra, Rodríguez y Artiles (2019), que presentan una experiencia muy interesante desarrollada también en el Grado de Educación Social, centran tanto el proyecto como la evaluación en el aprendizaje cooperativo, y éste desarrollado con todo el grupo-clase, y no por equipos de trabajo. La escala de satisfacción utilizada (que se puede deducir a partir del texto de la comunicación) se centra, por tanto, en este apartado. Por ello, tanto para el diseño del plan de evaluación como para los instrumentos específicos se han utilizado referentes propios.

La evaluación de contexto y la valoración del diseño inicial de la propuesta (evaluación de entrada, en términos de Stufflebeam) fue realizada fundamentalmente por el equipo docente, a partir de los aprendizajes adquiridos en la experiencia del curso anterior, contando también con aportaciones puntuales de estudiantes de ese mismo curso.

La evaluación de proceso recoge las valoraciones del profesorado a través de las reuniones de seguimiento, cuyo calendario inicial fue adaptado a la evolución real del proyecto en los diferentes grupos.

El alumnado también aporta información fundamental en esta evaluación de proceso. En septiembre se planteó una evaluación de expectativas en cada uno de los grupos (a través de una actividad por equipos en cada uno de los tres grupos-clase), y a primeros de noviembre se planteó una evaluación de seguimiento con dos técnicas complementarias de recogida de información: un cuestionario con preguntas cerradas sobre la evolución del trabajo, y una sesión presencial con cada grupo clase, diseñada a partir de la técnica de sombreros (De Bono, 2012), con una orientación claramente cualitativa. 
La evaluación de producto se suele centrar en los aprendizajes adquiridos, como se presentará en el apartado de resultados.

Pero en la evaluación de producto se ha incluido también, de una forma más detallada, la valoración del propio proyecto como estrategia didáctica, tanto desde una perspectiva cuantitativa (cuestionario individual de profesorado con escalas Likert cerradas) como cualitativa (cuestionario individual de profesorado con preguntas abiertas, y un grupo de discusión todavía no realizado) de la consecución de objetivos del proyecto y de elementos clave para su mejora (Jornet, González-Such y Perales, 2014).

En este sentido, la valoración de la perspectiva del alumnado también es fundamental, y contribuye a tener una visión más completa y holística del proyecto (Martínez Mediano, 2007). Para ello, se han utilizado también dos cuestionarios individuales (con preguntas cerradas, y abiertas) y una dinámica basada en técnicas creativas, planteada desde las artes plásticas, que ha permitido una discusión grupal más enriquecedora con el estudiantado (Perales, Cascales y García-Romeu, 2020; Catalá y Mesas, 2020).

Los cuestionarios se han diseñado para facilitar la triangulación de fuentes, de forma que se pudiera disponer de la perspectiva de alumnado y profesorado para las mismas cuestiones (evidentemente, adaptando la formulación de los ítems), y dejando espacios abiertos por bloques y al final para recoger otras valoraciones. En concreto, las dimensiones analizadas en estos dos cuestionarios son indicados en el Cuadro 1.

Cuadro 1. Instrumentos utilizados.

\section{Cuestionario de profesorado}

- Valoración global del trabajo interáreas (como producto y como proceso).

- Percepción de eficacia (consecución de los objetivos planteados en el proyecto de innovación docente, que son atribuibles al trabajo desarrollado hasta ahora)

- Percepción del valor didáctico de esta estrategia docente.

- Percepción de las dificultades encontradas por las y los estudiantes.

- Valoración de posibles mejoras que se han sugerido en momentos previos de evaluación del proyecto.

\section{Cuestionario de alumnado}

- Valoración global del trabajo interáreas (como producto y como proceso).

- Percepción de eficacia (consecución de los objetivos planteados en el proyecto de innovación docente, que son atribuibles al trabajo desarrollado hasta ahora)

- Percepción de las dificultades encontradas.

- Valoración de posibles mejoras que se han sugerido en momentos previos de evaluación del proyecto.

El proceso de evaluación no se ha cerrado. Está pendiente la realización de uno o varios grupos finales de discusión, donde poder mostrar los datos cuantitativos y cualitativos recogidos, y poder reflexionar conjuntamente sobre los mismos.

Otro colectivo implicado muy relevante serán las propias entidades que han acogido, acompañado, ayudado en la contextualización, y presentado una situación real a los equipos de trabajo de los estudiantes. Para el tercer trimestre está prevista una Jornada de Innovación Educativa, con su participación, para analizar esta experiencia e intentar optimizarla. Las propuestas de las propias entidades, a partir de la Jornada mencionada de Innovación Educativa, serán también fundamentales. 
Como se ha señalado en el apartado anterior, en ese trabajo nos centramos en la valoración final de estudiantado a partir de los resultados del cuestionario de preguntas cerradas.

\section{Resultados}

A continuación se presentan los resultados más significativos de cada uno de los apartados recibidos a partir de las valoraciones de estudiantes. Como se muestra en la tabla 1, la participación de estudiantes en el cuestionario cerrado ha superado globalmente el $30 \%$, siendo muy diferente entre los tres grupos-clase.

Tabla 1. Estadisticas de alumnado.

\begin{tabular}{ccc}
\hline Grupo & $\mathbf{N}$ & $\mathbf{\%}$ \\
\hline 1 & 5 & 7,1 \\
2 & 42 & 60,0 \\
3 & 23 & 32,9 \\
\hline
\end{tabular}

Los resultados, presentados a continuación, se organizan por bloques.

\subsection{Valoración global del trabajo interáreas.}

En un primer bloque se pregunta al alumnado sobre su valoración global del trabajo interáreas, utilizando una escala que varía de 1 - nada de acuerdo a 5 - muy de acuerdo. A continuación, en la Tabla 2 se ofrecen los indicadores con promedios y desviación típica.

Tabla 2. Promedios de las valoraciones finales: Valoración global.

\begin{tabular}{lcc}
\hline & Media & DT \\
\hline $\begin{array}{l}\text { Hacer el trabajo me ha ayudado a conocer mejor qué es un proyecto y cómo se debe abordar su } \\
\text { diseño }\end{array}$ & 4,47 & 0,812 \\
Hacer este trabajo ayudará a las estudiantes a la hora de abordar futuros trabajos durante el grado & 4,44 & 0,927 \\
$\begin{array}{l}\text { Considero que un trabajo como éste es necesario porque enriquece nuestra formación como } \\
\text { educadoras sociales }\end{array}$ & 4,36 & 0,817 \\
Considero que el esfuerzo ha merecido la pena & 4,19 & 1,026 \\
Estoy satisfecha con el resultado final del trabajo & 4,17 & 1,063 \\
$\begin{array}{l}\text { Considero que la nota del trabajo refleja de una forma justa la calidad final del trabajo en cada } \\
\text { parte }\end{array}$ & 3,81 & 1,04 \\
Las actitudes del profesorado han apoyado el desarrollo del trabajo & 3,56 & 0,987 \\
Los plazos globales establecidos en el trabajo se han podido cumplir & 3,47 & 1,1 \\
La presentación inicial de estudiantes de otros años nos ayudó a situarnos con el trabajo & 3,33 & 1,164 \\
Las estrategias utilizadas en clase han facilitado el desarrollo del trabajo & 3,33 & 0,896 \\
$\begin{array}{l}\text { El desarrollo del trabajo se ha ajustado a oportunidades o a imprevistos que han podido surgir a lo } \\
\text { largo del cuatrimestre }\end{array}$ & 3,24 & 0,955 \\
La presentación inicial del trabajo por parte del profesorado fue clara & 2,7 & 0,938 \\
\hline
\end{tabular}


En general, el alumnado valora de manera muy positiva los diferentes elementos del proyecto. Los indicadores mejor valorados se refieren a la percepción de utilidad del trabajo en cuanto al aprendizaje sobre el diseño de proyecto, su valor propedéutico y su vinculación con el desarrollo profesional. Se está valorando muy positivamente, por tanto, la pertinencia del proyecto. Se trata, además, de distribuciones bastante homogéneas (CV entre 18\% y 20\%). Otros ítems valorados por encima de 4 sobre 5 hacen referencia al esfuerzo invertido y el resultado conseguido (aunque aquí la variabilidad se incrementa un poco, CV 24,49 \% y 25,49 \%, respectivamente), siendo estos elementos también muy relevantes.

Como peor valorado ha sido señalado el indicador de claridad de la información presentada en la sesión inicial sobre el trabajo a realizar durante el curso $(2,7)$. Se realizó en una sesión conjunta del profesorado de cada grupo, ofreciendo una guía de trabajo concreta, pero con todo, como se verá más adelante, el arranque y concreción del trabajo ha sido uno de los elementos más costosos. La sesión complementaria realizada unas semanas más adelante, con estudiantes del curso anterior, fue valiosa, pero no tanto como el equipo docente habíamos previsto (3.33). Con todo, son las distribuciones más heterogéneas $(\mathrm{CV}=$ $34,74 \%$ y $34,95 \%$, respectivamente)

El resto de indicadores de la Tabla 2 han sido valorados bastante bien, por encima de los 3 puntos en una escala de 5, aunque destaca la diferencia entre su satisfacción respecto al trabajo final (4.17) y el ajuste percibido en la calificación recibida $(3,81)$. Probablemente, el grupo de discusión final, pendiente de realización, ofrecerá más elementos de análisis al respecto.

\subsection{Percepción de eficacia. Hasta qué punto se han cubierto los objetivos que nos planteábamos con el trabajo interáreas}

A continuación, en la Tabla 3 se ofrecen los indicadores y las puntuaciones medias del alumnado para esta dimensión, considerando que la escala utilizada fue de 1 - nada conseguido a 5 - totalmente conseguido.

Tabla 3. Promedios de las valoraciones finales: Alcance de objetivos planteados en el proyecto.

\begin{tabular}{lcc}
\hline \multicolumn{1}{c}{ Indicadores } & Media & DT \\
\hline Aprender a diseñar, elaborar, planificar y evaluar un PROYECTO de intervención & 4,23 & 0,871 \\
$\begin{array}{l}\text { Tomar como punto de partida para el diseño del proyecto una NECESIDAD REAL, del contexto o } \\
\text { de la entidad estudiada }\end{array}$ & 4,17 & 0,868 \\
Desarrollar técnicas colaborativas de TRABAJO EN GRUPO & 3,93 & 1,133 \\
$\begin{array}{l}\text { Facilitar la transdisciplinariedad y la COMPLEMENTARIEDAD del aprendizaje (un trabajo } \\
\text { abordado desde 5 asignaturas). }\end{array}$ & 3,86 & 0,997 \\
\hline
\end{tabular}

Desde los resultados presentados en la Tabla 3 se puede observar que el alumnado valora positivamente la consecución de los cuatro objetivos sobre los que se les pregunta. Valoran de una forma especialmente positiva la consecución de los objetivos más vinculados con sus competencias profesionales: aprender a diseñar, elaborar, planificar y evaluar un proyecto de intervención socio-educativa (4.23) y hacerlo a partir de una necesidad real identificada en un contexto (4.17).

Sin llegar, al 4 sobre 5, la valoración del desarrollo de técnicas colaborativas para trabajo en equipo es bastante elevada (3.93), pese a haber sido percibida por el profesorado, y haber manifestado el propio alumnado en las sesiones de valoración cualitativa que era un gran desafío (hay que señalar, no obstante, que es el ítem de este bloque donde hay mayor heterogeneidad en las valoraciones). Se puede deducir que, al final, valoran positivamente ese aprendizaje. 
La valoración más baja (aun siendo elevada) es la del objetivo de facilitar la transdisciplinariedad (3.86), es decir, no parece que se perciba como un objetivo totalmente alcanzado por el alumnado el haber conseguido la complementariedad de las cinco asignaturas presentadas en el proyecto.

En todos los casos, las distribuciones pueden ser calificadas de homogéneas (CV entre 20,59 y $28.53 \%$ )

\subsection{Valoración de las dificultades con las que se han encontrado durante el desarrollo del trabajo}

A continuación, en la Tabla 4 se ofrecen los indicadores y las puntuaciones medias para esta dimensión, que ha utilizado como escala la percepción de la dificultad que ha supuesto cada elemento para el alumnado (de 1 - poco difícil, a 5 - muy difícil).

Tabla 4. Promedios de las valoraciones finales: Elementos más importantes y las dificultades.

\begin{tabular}{lrr}
\hline \multicolumn{1}{c}{ Indicadores } & Media & DT \\
\hline La falta de tiempo para el desarrollo global del proyecto (no necesariamente en las clases) & 3,96 & 0,97 \\
La falta de tiempo durante las clases para hacer la parte vinculada con cada asignatura & 3,86 & 0,889 \\
La dificultad del grupo para quedar y para trabajar de modo eficiente & 3,41 & 1,222 \\
La dificultad intrínseca de cada una de las partes del trabajo & 3,09 & 0,794 \\
El apoyo de la entidad con la que queríamos trabajar & 2,8 & 1,431 \\
El apoyo real del profesorado implicado & 2,79 & 1,02 \\
Decidir cuál iba a ser nuestra aproximación al colectivo & 2,74 & 1,125 \\
La dificultad de llegar a acuerdos desde la discusión, y desde la valoración de todas las posturas & 2,54 & 1,2 \\
Decidir el tema y el colectivo con el que ibamos a trabajar & 2,53 & 1,176 \\
\hline
\end{tabular}

Desde la Tabla 4 podemos observar los resultados sobre las valoraciones de las dificultades y elementos más importantes. Así, se señalan como dificultades más relevantes las vinculadas con la falta de tiempo (global, en clase, y para el trabajo en grupo) y, en menor medida, la dificultad intrínseca de cada parte del trabajo.

La heterogeneidad de las distribuciones en los ítems relativos al trabajo en grupo (la dificultad para quedar, $\mathrm{CV}=35.84 \%$, y el establecimiento de acuerdo en el grupo, $\mathrm{CV}=47,24 \%$ ) confirma que los equipos de trabajo han funcionado de forma diferente, y eso se ha traducido en las percepciones sobre el propio desarrollo del trabajo. Estas valoraciones, también señaladas en las sesiones de trabajo del profesorado, y en las dinámicas cualitativas con estudiantes de proceso y de producto, que mostraban que unos grupos habían establecido un ritmo de trabajo adecuado y desde la corresponsabilidad, y en otros había desavenencias debidas en unos casos a diferencias de criterio a la hora de desarrollar el trabajo, y en otros casos a marcadas diferencias de implicación y responsabilidad.

La misma situación se produce respecto a la percepción de dificultad vinculada al apoyo de la entidad de referencia, siendo el ítem con mayor variabilidad $(\mathrm{CV}=51,11 \%)$. La acogida y el apoyo de las entidades han condicionado, por tanto, la dificultad percibida por los equipos de estudiantes.

En el otro extremo, se manifiesta una menor dificultad percibida respecto a las decisiones iniciales respecto al trabajo, el tema y el colectivo, y la aproximación específica, aunque ambos ítems muestran, nuevamente, distribuciones heterogéneas ( $\mathrm{CV}$ de 46,48 \% y 41,06\%, respectivamente). 
Por otro lado, en la aproximación cualitativa del cuestionario el estudiantado ha señalado los siguientes elementos como importantes:

- Cambio de formato de trabajo: dificultades experimentadas en el comienzo, contacto inicial con entidades o la primera toma de contacto con el trabajo, ya que no sabíamos muy bien por dónde tirar, dificultad de encontrar un centro, necesidad de recurrir a más de un centro.

- Organización docente: el apoyo e implicación por parte de algún/a docente, falta de atención, poca implicación del profesorado en alguna asignatura; poca cohesión entre lo teórico y el contexto real.

- Organización del alumnado: escasa participación de los miembros del grupo, la existente competitividad entre los grupos, y en muchas ocasiones se restan información (de unos grupos a otros), la dificultad a la hora de exponer el proyecto de forma creativa, la escasa participación de alguna o algún miembro del grupo, la falta de escucha activa durante la realización del trabajo, la falta de entendimiento y empatía por parte del grupo de trabajo a la hora de quedar para realizar el trabajo, la incompatibilidad de las componentes del grupo, las ganas de cada miembro del grupo con el trabajo y tema.

- Otros: la dificultad de comunicar nuestro estrés;

\subsection{Posibles mejoras}

En las otras dinámicas de evaluación con alumnado (de proceso y de producto) de tipo cualitativo, se han aportado algunas sugerencias. En este momento se ha considerado relevante contrastas estas propuestas con los dos colectivos (profesorado y alumnado, con la escala: 1 es una propuesta poco adecuada, 5 es una propuesta muy adecuada). A continuación, en la Tabla 5 se señalan las valoraciones del estudiantado.

Tabla 5. Promedios de las valoraciones finales: Posibles mejoras.

\begin{tabular}{|c|c|c|}
\hline Indicadores & Media & DT \\
\hline $\begin{array}{l}\text { Sería interesante disponer de un listado inicial de entidades propuestas, que conocieran la } \\
\text { iniciativa y nos pudieran apoyar, aunque eso redujera nuestro margen de elección }\end{array}$ & 4,33 & 0,88 \\
\hline $\begin{array}{l}\text { Sería interesante implementar el proyecto en los centros, aunque eso añadiera más estrés al } \\
\text { proyecto }\end{array}$ & 3,39 & 1,266 \\
\hline $\begin{array}{l}\text { Sería interesante que los grupos se organizaran de forma aleatoria, para ayudarnos a trabajar } \\
\text { con personas nuevas }\end{array}$ & 2,44 & 1,471 \\
\hline
\end{tabular}

En general, el alumnado ve necesario recibir un listado de entidades con las que podrían trabajar, y son especialmente de acuerdo en ello.

La posibilidad de implementar el proyecto que se diseña surgió también en las sesiones cualitativas de trabajo con estudiantado, porque reclamaban reforzar la perspectiva práctica, aplicada, del trabajo; y porque valoraban muy positivamente el contacto inicial que tuvieron con los contextos y las entidades para hacer el análisis de necesidades. Sin embargo, la valoración de esta posibilidad supera un poco el punto medio de la escala, pero la distribución es bastante heterogénea $(\mathrm{CV}=37.34 \%)$. El estrés que añadiría esta opción, y la falta de tiempo real para llevarlo a cabo, argumentos que surgieron en la evaluación cualitativa, pueden explicar estos datos.

En último lugar, la posibilidad de formar los grupos de forma aleatoria es la que recibe una valoración inferior, y además con una distribución muy heterogénea $(\mathrm{CV}=68,29)$. También en la sesión de cierre 
cualitativa, y en la sesiones de profesorado, se analizó este tema, sus ventajas e inconvenientes, sin llegar a conclusiones claras.

Además, desde una perspectiva cualitativa, el alumnado ha señalado otras propuestas:

- Hablar los conflictos que puedan surgir en el grupo a la hora de trabajar, ya sea de forma individual con el grupo o en clase de forma general.

- Una mayor retroalimentación sobre el trascurso de trabajo por parte del profesorado y también más tiempo para llevar a cabo el proyecto.

- Organizar las entregas de trabajos de cada asignatura en distintas fechas, para poder terminar un punto después de otro y focalizar mejor la atención al no llevar tantas cosas al mismo tiempo.

Finalmente, las y los estudiantes han aportado comentarios generales, que presentamos a continuación, de manera agrupada:

- La opiniones sobre que el proyecto debe ser anual, debido a su gran volumen y magnitud de trabajo; además, si se cuenta con la implementación del proyecto grupal en las entidades colaboradoras, sería interesante, pero añadiría más estrés, no llegaría a implementarse totalmente (sería por partes) por la falta de tiempo, ya que la temporalización del cuatrimestre se percibe como bastante escasa para ese fin.

- Visiones positivas y negativas sobre la composición aleatoria de los componentes del grupo. Negativas: falta de posibilidad de compaginar las reuniones por trabajo; positivas: las personas que tienen diferentes asignaturas (no están matriculadas en todas las asignaturas del curso) deben poder participar en el diseño del proyecto.

- Sobre la organización docente: se solicita la alternativa de trabajo para aquellas personas que ya trabajan en la intervención. Se valora el trabajo en este proyecto como valioso, aunque difícil (sobre todo al principio). Se recalca la necesidad de mejor organización en cuanto a las rúbricas de evaluación del proyecto y la necesidad de tener un listado de centros o entidades que están dispuestas a colaborar. Se propone no hacer exámenes, dado que con la elaboración del interareas se trabaja y aprende suficiente.

\section{Conclusiones}

La transdisciplinariedad como medio facilitador de la construcción de aprendizajes de manera autónoma y compartida se muestra como una herramienta de utilidad para el aprendizaje. Aunando la teoría y la práctica se permite el aprendizaje significativo del alumnado del Grado de Educación Social de la Universidad de Valencia.

Además, el proyecto interáreas, como tarea específica transdiciplinar, ha permitido tener una mirada globalizadora de diversas áreas de aprendizaje que, trabajadas de manera conjunta, han permitido la interrelación de aprendizajes. Todo ello teniendo en cuenta el desafío que supone en la actualidad el proceso de enseñanza- aprendizaje en el ámbito universitario tanto por parte del alumnado como del profesorado (Perales, Conchell, De Fez, Bellver y Horcas, 2020).

El aprendizaje por proyectos no es una metodología innovadora ya que se viene desarrollando tanto en educación primaria y secundaria cada vez de manera más regular y en el ámbito universitario, como se ha visto anteriormente, se incorpora de manera más lenta pero con paso firme (Fernández-Cabezas, 2017), con experiencias también muy interesantes que integran la dimensión de aprendizaje colaborativo, desde el término de "aprendizaje por proyectos colaborativos" (Maldonado Pérez, 2008). 
En este caso, pensamos que la fuerza y la innovación de la metodología presentada vienen dadas por el trabajo desde diversas asignaturas, y por la implicación conjunta de todas las personas que intervienen en el mismo: alumnado, profesorado y centros de trabajo. Además, el proyecto se refuerza porque es reconocido institucionalmente como proyecto de innovación educativa dentro de la propia Universidad.

Como se ha indicado, pensamos que la evaluación es un elemento fundamental en esta metodología transdisciplinar y que mediante las herramientas propuestas para la misma, tanto cuantitativas como cualitativas, durante todo el proceso obtendremos la información necesaria que nos ayude a medir el cumplimiento de objetivos. Además, las mismas herramientas se convierten en herramientas didácticas, pues son trabajadas en clase y podrán ser utilizadas por el alumnado como profesionales de la Educación Social.

Respecto a los resultados concretos, en síntesis, queremos señalar lo más importante.

La valoración general del proyecto por parte del alumnado es muy positiva, utilidad del trabajo en cuanto al aprendizaje sobre el diseño de proyecto, su valor propedéutico y su vinculación con el desarrollo profesional Se señala la falta de entendimiento en la presentación del proyecto interáreas, lo que es comprensible, ya que es un proyecto que anteriormente no han realizado en su trayectoria estudiantil. En general, además, en los comentarios se observa la falta de costumbre de trabajo autónomo y autoorganizado por parte del alumnado, que demanda mayor cantidad de regulación y normas (evaluación más concreta, temporización de tareas, seguimiento más estrecho por parte docente, etc.).

Entre los elementos más importantes que influyen en el trabajo y pueden causar el estrés se señala la falta de tiempo para desarrollar el trabajo grupal. Es importante que la mayor parte de dificultades señaladas ha sido sobre la falta de participación de los mismos componentes de grupos, en muchas ocasiones el alumnado tiene falta de iniciativa y una escasa voluntad en las tareas grupales.

Respecto a los objetivos planteados en el proyecto, se puede señalar que la cantidad y calidad de trabajos entregados, la valoración cualitativa y cuantitativa realizada durante el seguimiento y las dinámicas de evaluación final realizadas aportan evidencias respecto a su consecución (Perales, Conchell, y Lorente, 2020).

En concreto, a partir de a las evidencias derivadas de las valoraciones de las y los estudiantes, respecto a la consecución de los objetivos del proyecto podemos destacar lo más significativo.

Las valoraciones aportadas por el estudiantado en los diferentes bloques de cuestiones muestran que los objetivos 2 y 4 (referidos a centrar la estrategia didáctica en el diseño de un proyecto de intervención, y hacerlo a partir de la identificación de una necesidad real, en un contexto específico) pueden considerarse claramente conseguidos. La referencia al diseño de un proyecto profesional, y a su vinculación una necesidad real identificada en un contexto concreto, son reiteradamente puestos en valor por el alumnado, de forma similar al profesorado.

El objetivo 3, relativo a las técnicas cooperativas de trabajo en equipo, es percibido como conseguido por el alumnado. Aunque no tiene una valoración tan alta como los objetivos 2 y 4 , se mantiene en un nivel muy elevado (3.93 sobre 5). La identificación de dificultades, sin embargo, muestra que esta valoración depende mucho del funcionamiento de cada equipo de trabajo, y por tanto debería ser abordad de una forma más explícita, aplicando estrategias de aprendizaje cooperativo.

El objetivo 1, relativo a la transdisciplinariedad del trabajo, es el percibido como conseguido con menor intensidad por el alumnado, aunque se mantiene en un nivel alto (3.86). Se ha desarrollado un intenso trabajo de coordinación por parte del equipo docente, previo y durante el desarrollo del proyecto, pero es necesario seguir trabajando en ello. 
En cuanto a los objetivos 5 y 6 , se refieren al trabajo del equipo docente, y los estudiantes no han sido consultados respecto a ellos.

El objetivo 5 se considera conseguido, considerando el plan de evaluación desarrollado. Este plan se ha planteado desde una perspectiva integral y ambiciosa, con diferentes criterios, fuentes, momentos y técnicas de recogida de información, trabajando desde el modelo de complementariedad metodológica (Perales, Ortega y Jornet, 2011; Perales, Cascales y García-Romeu, 2020).

Pero es un objetivo no finalizado. Todavía hay mucha información pendiente de ser analizada de una forma sistemática, abordando diferentes estrategias de triangulación. Además, mediante una estrategia de metaevaluación, plantearemos la revisión del propio plan de evaluación y de sus instrumentos, tanto desde los criterios de utilidad, legitimidad, precisión, viabilidad y responsabilidad (Joint Committee on Standards for Educational Evaluation, 2010) como desde los de equidad, justicia y utilidad (Jornet, Suárez y Perales, 2003).

Para el objetivo 6, como se marca en la comunicación paralela (Perales, Conchell y Lorente, 2020, s.p.):

Hasta el momento se han presentado, con éste, tres trabajos en congresos de innovación educativa, con un gran esfuerzo de sistematización de la información. La consecución de este objetivo, de todas formas, sigue abierta, y será necesario analizar, paulatinamente, la información recogida.

En este mismo sentido, para la difusión de resultados, y para la mejora del propio proyecto, se está organizando para la primera semana de mayo una Jornada de transferencia de conocimiento con las entidades que han colaborado (este curso, y los anteriores) en este tipo de trabajos. El objetivo, en términos de difusión, será presentar el trabajo realizado, y el valor para el aprendizaje de las y los estudiantes. Pero también, avanzar hacia una consolidación de la relación iniciada, que permita optimizar la identificación y atención de necesidades, formalizar un listado de entidades dispuestas a participar de forma activa, que sea un buen punto de arranque para las y los estudiantes, y también contribuir a la mejora de la intervención en el mundo profesional.

De acuerdo con todo lo expuesto, las valoraciones del estudiantado confirman la pertinencia y relevancia de un proyecto como éste, aunque ponen de manifiesto elementos que deben ser mejorados. En los apartados cualitativos de los cuestionarios, varias personas han señalado la necesidad de ampliar la temporalización de este proyecto, hacerlo anual; de esta manera sería más viable llevar el proyecto grupal a la práctica y observar cómo se puede desarrollar en las condiciones reales. Esto, sin embargo, es difícilmente viable dentro de la estructura académica. Por otra parte, el alumnado señala que en general se podría evitar el estrés mediante un mayor apoyo por parte del profesorado, una mejor organización documental (listado de entidades, rúbricas de evaluación, retroalimentación) más tiempo para trabajo grupal, y la organización de fechas de entrega distintas para diferentes asignaturas. Todas estas propuestas deberán ser analizadas para la próxima edición del proyecto.

Para terminar, resaltamos la importancia que da el alumnado a tener un acercamiento al mundo profesional para su formación de Grado resignificando aprendizajes teóricos llevados a la práctica y teniendo en cuenta la realidad de cada uno de los contextos trabajados. Por otro lado, la dinámica final de socialización de proyectos desarrollados valoriza el trabajo desarrollado, contribuye al desarrollo de técnicas profesionales de exposición pública, y contribuye al aprendizaje compartido, viendo los proyectos desarrollados por todos los equipos de cada grupo-clase. La presencia de todo el profesorado de cada grupo en esas sesiones, y el feedback colegiado ofrecido, cierra el proceso con el alumnado desde una apuesta clara por la coordinación docente. Lo cierra... aparentemente. Queda abierto para seguir 
analizando en estos meses, para reflexionar sobre los elementos a mejorar y las estrategias posibles, y para diseñar la propuesta para el próximo curso.

\section{Referencias}

ALFONSO-BENLLIURE, V. y BELLVER, M.C. (2020). "Creatividad: un reto en la educación superior" en Bellver, M.C., Verde, I. (coord.). Educación social y creatividad. Fundamentación, estrategias de intervención y experiencias desde diferentes lenguajes artísticos (pp. 17-41). Valencia: Tirant lo Blanch.

CATALÁ, E. y MESAS, E.C. (2020). "Las artes plásticas como estrategia para la intervención en la educación social” en Bellver, M.C., Verde, I. (coord.). Educación social y creatividad. Fundamentación, estrategias de intervención y experiencias desde diferentes lenguajes artísticos (pp. 227-247). Valencia: Tirant lo Blanch.

DE BONO, E. (2012). Seis sombreros para pensar. Madrid: Paidós.

FERNÁNDEZ-CABEZAS, M. (2017). “Aprendizaje basado en proyectos en el ámbito universitario: una experiencia de innovación metodológica en educación" International Journal of Developmental and Educational Psychology, vol. 2, issue 1, pp. 269-278. <https://doi.org/10.17060/ijodaep.2017.n1.v2.939>

FONT, A. (2004). "Líneas maestras del aprendizaje por problemas" en Revista interuniversitaria de formación del profesorado, vol. 18, issue 1, pp. 79-95.

GUERRA SANTANA, M. RODRÍGUEZ PULIDO, J. y ARTILES RODRÍGUEZ, J. (2019). “Aprendizaje colaborativo: experiencia innovadora en el alumnado universitario" en Revista de Estudios y Experiencias en Educación, vol. 18, issue 36, pp. 269-281. <http://dx.doi.org/10.21703/rexe.20191836guerra5>

GROS, B. y LARA, P. (2009). "Estrategias de innovación en la educación superior: el caso de la Universitat Oberta de Catalunya" en Revista Iberoamericana de Educación, vol. 49, issue 1, pp. 223-245.

JOINT COMMITTEE ON STANDARDS FOR EDUCATIONAL EVALUATION (2010). The program evaluation standards. California: Sage.

JORNET, J., GONZÁLEZ-SUCH, J. y PERALES, M. J. (2014). Investigación evaluativa. Una perspectiva basada en la complementariedad metodológica (cuantitativa-cualitativa). Valencia-Lima: Universitat de València e IESP CREA.

JORNET, J.M., SUÁREZ, J.M. y PERALES, M.J. (2003). Guía práctica para la evaluación de programas de formación profesional, ocupacional y continua. Valencia: Adeit.

MALDONADO PÉREZ, M. (2008). “Aprendizaje basado en proyectos colaborativos. Una experiencia en educacion superior" en Laurus, vol. 14, issue 28, pp. 158-180.

MARTÍNEZ MEDIANO, C. (2007). Evaluación de programas: modelos y procedimientos. Madrid : UNED

MORALES, P. y LANDA, V. (2004). “Aprendizaje basado en problemas” en Theoria,13, pp. 145-157.

NAVARRO, L. P. (2006). "Aprendizaje activo en el aula universitaria: el caso del aprendizaje basado en problemas" en Miscelánea Comillas. Revista de Ciencias Humanas y Sociales, vol. 64, issue 124, pp. 173-196.

PERALES, M.J., CONCHELL, R., DE FEZ, S., BELLVER, M.C. y HORCAS, V. (2020) [en revisión]. "Projec3es. El diseño transdisciplinar de un proyecto de intervención como herramienta de aprendizaje significativo en el tercer curso del grado en educación social de la Universitat de València” En VIII Congreso Estatal de Educación Social. Educacion social, dignidad y derechos. Zaragoza.

PERALES, M. J., ORTEGA, S. y JORNET, J. (2012). "La evaluación como condición de calidad en la educación intercultural" en Díe, L. Aprendiendo a ser iguales. Manual de Educación Intercultural. Valencia: CeiMigra.

PERALES, M.J., CASCALES, J. y GARCÍA-ROMEU, B. (2020). "Evaluando con creatividad: técnicas creativas para la evaluación de programas socioeducativos" en Bellver, M.C., Verde, I. (coord.) Educación social y 
creatividad. Fundamentación, estrategias de intervención y experiencias desde diferentes lenguajes artísticos (pp. 365-398). Valencia: Tirant lo Blanch.

PERALES, M.J., CONCHELL, R. y LORENTE, M. (2020) [en revisión]. "El Proyect3ES como metodología transdisciplinar de aprendizaje por proyectos en el Grado de Educació Social. La valoración del profesorado. En $I N$ RED 2020. VI Congreso de Innovación Educativa y Docencia en Red. Valencia.

PÉREZ JUSTE, R. (2014). Evaluación de programas educativos. Madrid : La Muralla.

STUFFLEBEAM, D. y SHINKFIEL, A.J. (1987). Evaluación sistemática. Madrid: Paidós.

VERDE, I. y BELLVER, M.C. (2020). "Creatividad e innovación, promoviendo el cambio" en Bellver, M.C., Verde, I. (coord.) Educación social y creatividad. Fundamentación, estrategias de intervención y experiencias desde diferentes lenguajes artisticos (pp. 43-69). Valencia: Tirant lo Blanch. 